\title{
Article \\ Biosensor for Detecting Fetal Growth Restriction in a Low-Resource Setting
}

\author{
Anders Jacobsen ${ }^{1}$, Christentze Schmiegelow ${ }^{2}$, Bjarke Sørensen ${ }^{3}$, Omari A. Msemo ${ }^{4}$, Karsten Nielsen ${ }^{5}$, \\ Birgitte Bruun Nielsen ${ }^{6}$, Sofie Lykke Møller ${ }^{7}$, John P. A. Lusingu ${ }^{2,4}$, Daniel T. R. Minja ${ }^{4}$ (D), Morten Hedegaard ${ }^{8}$ \\ and Diana Riknagel ${ }^{9, *}$
}

\section{check for} updates

Citation: Jacobsen, A.; Schmiegelow, C.; Sørensen, B.; Msemo, O.A.;

Nielsen, K.; Nielsen, B.B.; Møller, S.L.; Lusingu, J.P.A.; Minja, D.T.R.;

Hedegaard, M.; et al. Biosensor for Detecting Fetal Growth Restriction in a Low-Resource Setting. Reprod. Med. 2021, 2, 57-67. https://doi.org/ $10.3390 /$ reprodmed 2010007

Academic Editor: Berthold Huppertz

Received: 14 January 2021

Accepted: 15 February 2021

Published: 15 March 2021

Publisher's Note: MDPI stays neutral with regard to jurisdictional claims in published maps and institutional affiliations.

Copyright: (C) 2021 by the authors Licensee MDPI, Basel, Switzerland. This article is an open access article distributed under the terms and conditions of the Creative Commons Attribution (CC BY) license (https:// creativecommons.org/licenses/by/ $4.0 /)$.
1 Illulissat Hospital, 3952 Illulissat, Denmark; anzelz@hotmail.com

2 Center for Medical Parasitology, Department of Immunology and Microbiology, University of Copenhagen, 2200 Copenhagen, Denmark; chsch@sund.ku.dk (C.S.); jpalusingu@gmail.com (J.P.A.L.)

3 Department of Obstetrics and Gynecology, Zealand University Hospital, 4000 Roskilde, Denmark; blsr@regionsjaelland.dk

4 National Institute for Medical Research, Tanga Center, P.O. Box 5004, Tanga, Tanzania; mtengeti@gmail.com (O.A.M.); minjartd@gmail.com (D.T.R.M.)

5 Institute of Pathology, Aarhus University Hospital, 8200 Aarhus, Denmark; karsni@rm.dk

6 Department of Obstetrics and Gynecology, Institute of Clinical Medicine, Aarhus University, 8200 Aarhus, Denmark; birgitte.bruun.nielsen@dadlnet.dk

7 Department of Public Health, Global Health Section, University of Copenhagen, 1017 Copenhagen, Denmark; sofiem@sund.ku.dk

8 Hedegaard Clinic, 2100 Copenhagen, Denmark; morten.hedegaard@dadlnet.dk

9 Department of Obstetrics and Gynecology, Regional Hospital Viborg, 8800 Viborg, Denmark

* Correspondence: Diana.Kjaer.Riknagel@Viborg.RM.dk

Abstract: One strategy for improving detection of fetal growth restriction (FGR) is developing biosensors identifying placental dysfunction as a leading pathogenesis for FGR. The aim of this pilot study was to investigate the performance of a biosensor specified to detect placental dysfunction by means of maternal arterial turbulence acoustics in a low-resource setting. A cohort of 147 singleton pregnant women were prospectively followed with double-blinded biosensor tests, sonographic estimation of fetal weight (EFW) and Doppler flow at 26-28, 32-34 and 37-39 weeks of pregnancy. Full term live births with recorded birth weights (BWs) and without major congenital malformations were included. Outcomes were defined as (A) a solitary biometric measure (BW < 3rd centile) and as (B) a biometric measure and contributory functional measure $(\mathrm{BW}<10$ th centile and antenatally detected umbilical artery pulsatility index $>$ 95th centile). Data from 118 women and 262 antenatal examinations were included. Mean length of pregnancy was 40 weeks (SD \pm 8 days), mean BW was $3008 \mathrm{~g}$ (SD $\pm 410 \mathrm{~g})$. Outcome (A) was identified in seven (6\%) pregnancies, whereas outcome (B) was identified in one $(0.8 \%)$ pregnancy. The biosensor tested positive in five $(4 \%)$ pregnancies. The predictive performance for outcome $(\mathrm{A})$ was sensitivity $=0.29$, specificity $=0.97, p=0.02$, positive predictive value (PPV) was 0.40 and negative predictive value (NPV) was 0.96 . The predictive performance was higher for outcome (B) with sensitivity $=1.00$, specificity $=0.97, p=0.04, \mathrm{PPV}=0.20$ and NPV $=1.00$. Conclusively, these pilot-study results show future potential for biosensors as screening modality for FGR in a low-resource setting.

Keywords: fetal growth restriction; intrauterine growth restriction; placental dysfunction; maternal vascular malperfusion; uteroplacental blood flow; prenatal diagnostics; antenatal screening

\section{Introduction}

The clinical effort of identifying fetal growth restriction (FGR) is substantial [1] and crucial as FGR is associated with more than half of all still-births as well as long-term health with increased risk of cardiovascular disease for survivors [1-3]. A significant change in perspective is shifting the clinical focus from screening for FGR using the lowest biometric 
measures (e.g., fetal weight) of a population-based or customized reference [3] to using various biomarkers of placental dysfunction [4]. In parallel, the development of healthcare communication architecture provides new opportunities for biosensors and remote health monitoring independent of clinical setting and expertise [5].

Recently, an acoustic approach for the identification of FGR secondary to placental dysfunction has been investigated, and found to be significantly associated with FGR, but not with small-for-gestational-age (SGA) fetuses [6-8]. A biosensor consisting of wearable mini-microphones is patched wirelessly to the skin of the lower abdomen, medially to the uterine arteries for capturing maternal vascular sounds emanating locally from beneath the sensors. The vascular sound characteristics obtained by the biosensor can be segmented into different sound frequency spectrums for visual and automatically inspection. Hereby, contents will reveal cardiac valve sounds in one frequency spectrum (maternal and fetal), and local arterial pulse sounds in another frequency spectrum [6-8]. Empirical studies on partly occluded arteries, as in cases of coronary artery stenosis, show that acoustic turbulent blood flow appears in a distinct frequency spectrum $>200 \mathrm{~Hz}$ [9]. Normally, blood flows in a streamlined profile with its highest velocity in the center of the streamline as blood cells are held together by viscous forces. If the blood stream enters an area with reduced diameter the velocity increases, and if the velocity becomes too high the kinetic energy overcomes the viscous energy and causes the streamlined profile to break apart and become random and chaotic-turbulent. Laminar flow in arteries is silent whereas turbulent blood flow creates pulse-sounds clinically known as arterial murmurs $[9,10]$. Findings on the physiological properties of blood flow in relation to abnormal placentation show that the inflow of maternal blood into the intervillous space (IVS) is indeed turbulent [11], and velocity is increased by a factor 10-20 compared to that of normal placentation [12]. The maternal high-velocity turbulent jets creating vortices of inflow in the intervillous space are the main contributors to the endothelial shear-stress injuries related to placental lesions and subsequent manifestation of FGR [12-14]. This micro-rheological change in the IVS cannot currently be identified clinically. The mechanism is previously investigated by modeling [11,12], and now by interpretation of acoustic characteristics of the maternal arterial pulse obtained near the uterine arteries.

The aim of this pilot study was to investigate the applicability of a biosensor specified to detect placental dysfunction by means of maternal arterial turbulence acoustics in a low-resource setting.

\section{Materials and Methods}

A sub-group of singleton pregnant women in the FOetal exposure and Epidemiological Transition: the role of Anemia in early life for Non-Communicable Diseases in later life (FOETALforNCD) cohort from Tanga Region, northeastern Tanzania was recruited for this sub-study [15]. The FOETALforNCD study was an interdisciplinary study of 1415 preconception women and 538 pregnant women to investigate the role of anemia in early life for non-communicable diseases (NCDs) in later life [15]. From May to November 2016, a total of 147 participants were recruited during an antenatal examination in pregnancy weeks 26-28 (+/ - one week) or in 32-34 (+/ - one week) at Korogwe District Hospital $(\mathrm{KDH})$. If included in pregnancy weeks $26-28(+/-$ one week), participants were followed in weeks 32-34 (+/ - one week) and in weeks 37-39 (+/ - one week). If included in weeks 32-34 (+/ - one week), they were also examined in weeks 37-39 (+/ - one week), (Figure 1). 


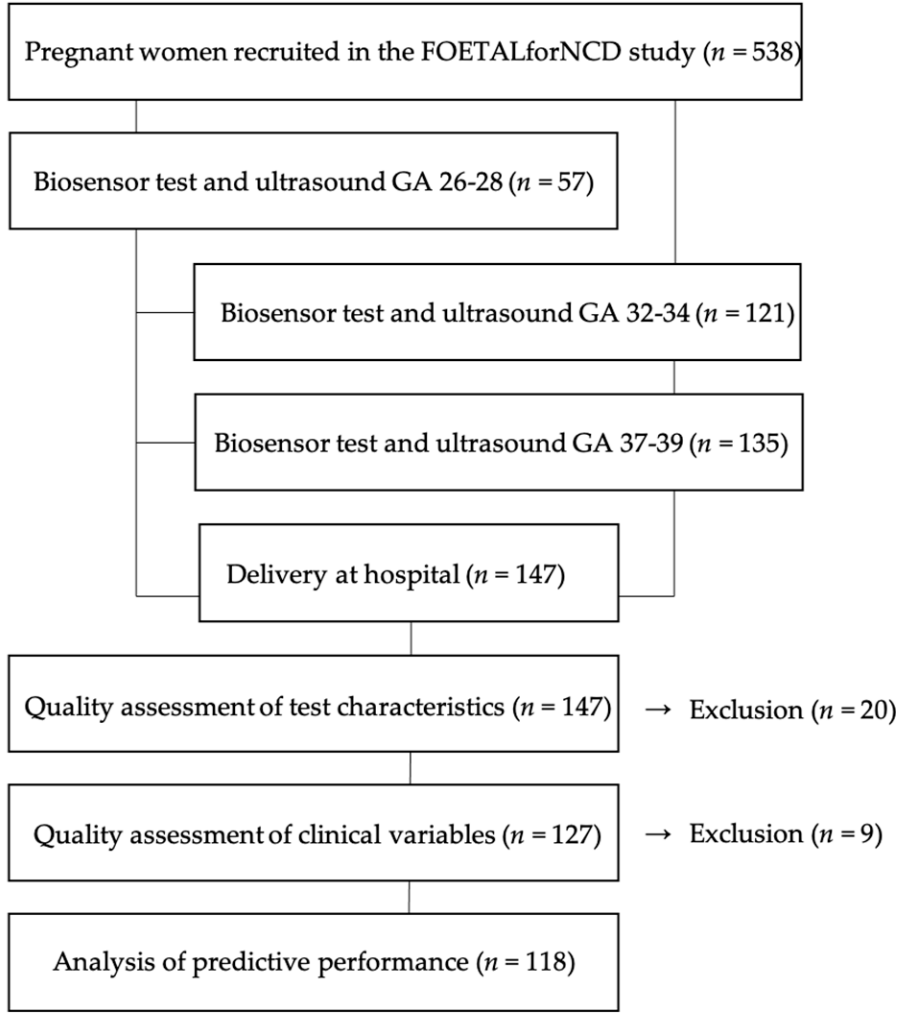

Figure 1. Flow chart of inclusion, intervention, exclusion and analyses in the study.

Inclusion criteria for the sub-study were singleton- and ultrasound-dated pregnancy before 21 weeks of pregnancy. Exclusion criteria were missing biosensor test and ultrasound at the same antenatal examination, biosensor test quality assessment score below 3 in a quality index ranging 1-4, described in detail elsewhere [6-8], birth before full 37 weeks, major congenital malformations, stillbirth or missing BW. Of the 147 women included, $20(14 \%)$ were excluded due to low quality assessment of the sound characteristics, and nine $(6 \%)$ women were excluded due to clinical reasons whereof seven had missing clinical data, one preterm birth and one with major congenital malformations.

Clinical data included gestational age (GA) based on crown-rump length measured in the first trimester and head circumference measured in the second trimester using the Intergrowth-21st algorithm [16,17], maternal blood pressure, pulse, hemoglobin level, estimated fetal weight (EFW), umbilical artery (UA) Doppler, uterine artery (UtA) Doppler, BW (BW) and placenta weight.

Biosensor data consisted of a three-minute, double-blinded sound recording obtained transcutaneous with two microphones (microphone 4060, Danish Pro Audio microphones, Alleroed, Denmark) attached wirelessly to the abdominal skin above the pelvic bone and medially from the umbilicus using a dedicated adhesive medical grade patch (Nile AB, Ängelholm, Sweden) covering the abdominal curve between the bilateral anterior superior iliac spines (Figure 2). 


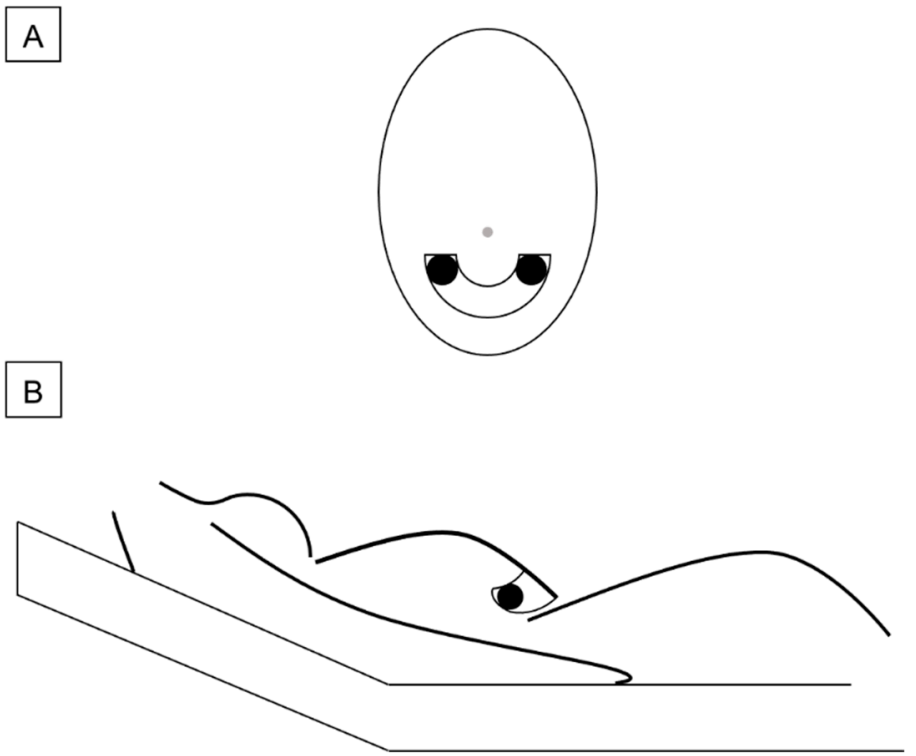

Figure 2. Illustration of (A) the biosensor containing two microphones attached medially from the umbilicus using a dedicated adhesive medical grade patch covering the abdominal curve between the bilateral anterior superior iliac spines, and (B) the pregnant woman lying in a semi-recumbent position while being monitored by the biosensor.

The biosensor data were masked during recording and transferred to computerstorage for blinded quality analysis. Raw sound signals from each microphone-channel were assessed independently for signal-to-noise ratio in six $60 \mathrm{~s}$ segments per recording. The segments were rated in a quality index ranging from one to four in the cardiac-valve frequency spectrum below 100 hertz $(\mathrm{Hz})$ and in the arterial turbulence frequency spectrum above $200 \mathrm{~Hz}$ (Table 1). A quality score of one in the cardiac-valve frequency spectrum was given to data with excellent signal quality showing systolic-diastolic periods without physiological (e.g., bowel-movements) or ambient noise. A quality score of two in the cardiac-valve frequency spectrum was given if data showed same content as in quality score of one, but with some degree of physiological or ambient noise. The quality scores of three and four were given when the signal in the cardiac-valve frequency spectrum contained noise artefacts either blurring the systolic-diastolic periods (score of three), or without systolic-diastolic periods (score of four), (Table 1).

Table 1. Number of sound quality rates assessed in $60 \mathrm{~s}$ segments for each microphone channel distributed in a cardiac-valve frequency spectrum $<100 \mathrm{~Hz}$, and in an arterial turbulence frequency spectrum $>200 \mathrm{~Hz}$.

\begin{tabular}{ccc}
\hline Quality Index & Cardiac Valve Frequency $^{\mathbf{1}^{\mathbf{1}}}$ & Arterial Turbulence Frequency $^{\mathbf{1}}$ \\
\hline 1 & 390 & 294 \\
2 & 824 & 1022 \\
3 & 472 & 388 \\
4 & 192 & 174 \\
Total $(n)$ & 1878 & 1878 \\
\hline
\end{tabular}

${ }^{1}$ Number of segments.

In the arterial turbulence frequency spectrum, all segments were rated according to the following: quality index score of one was given if the segment contained no physiological or ambient noise. A score of two was given if the segment contained some sporadic noise. A score of three was given if consistent noise was present, and a score of four was given if the segment was blurred with noise in all $60 \mathrm{~s}$. All segments in the quality index scores of four were excluded to ensure homogeneity in the data used for interpretation. If more than three $(50 \%)$ of each microphone recording was indexed with a score of three or four the 
entire recording was excluded. A total of 313 tests containing two-channel microphone recordings of 1878 sound segments analyses was performed (Table 1). Hereby, 20 study subjects were excluded.

Each included segment in the arterial turbulence frequency-spectrum was finally dichotomously classified as either containing a persistent systolic high-frequency energy defined as a maternal arterial turbulence (MAT+, Figure 3) or containing no maternal arterial turbulence (MAT-, Figure 4). After classification the sound segments were assessed per subject, enabling detection of unilateral classification if only one of the bilateral-positioned microphones obtained MAT+ recordings, or bilateral classification in cases of both microphones obtained MAT+ recordings.
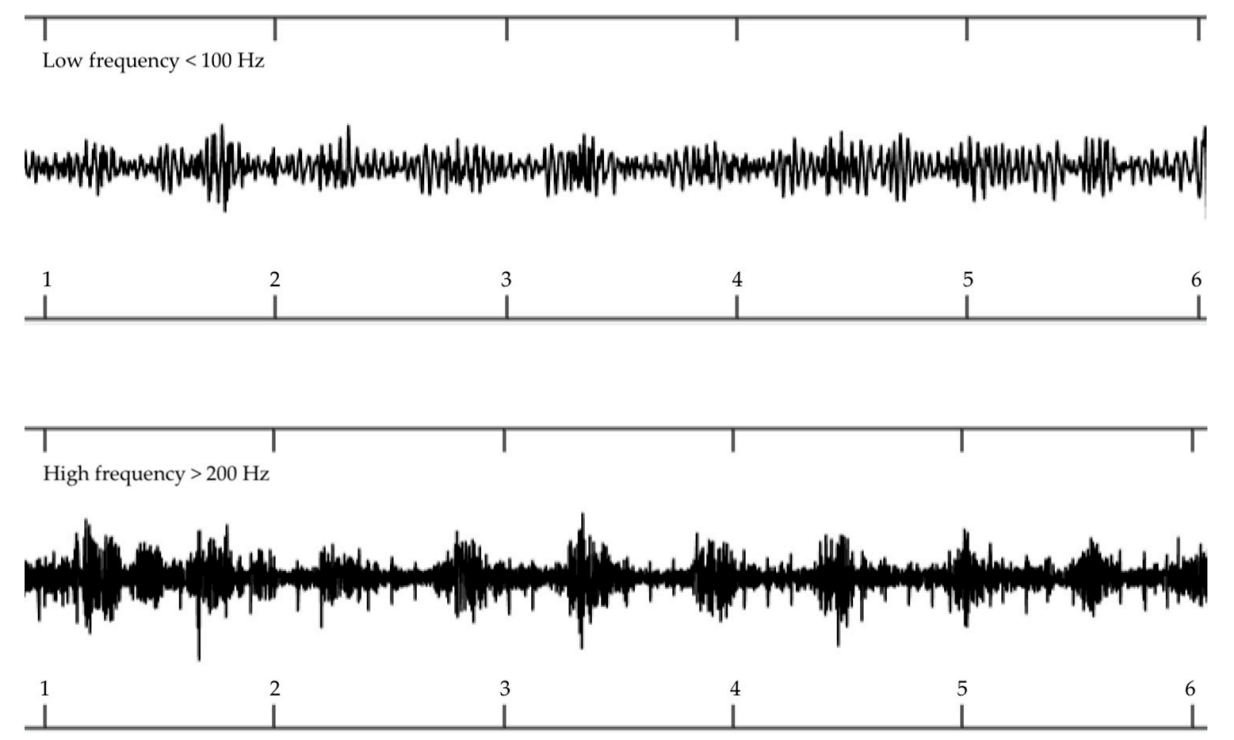

Figure 3. Example of a maternal arterial turbulence (MAT+) test illustrating in the upper graph a cardiac-valve frequency spectrum below $100 \mathrm{~Hz}$, and in the lower graph an arterial turbulence frequency spectrum above $200 \mathrm{~Hz}$ with a clear visible pulsatile pattern. The $Y$-axis shows time in seconds. The amplitude is shown in arbitrary units.

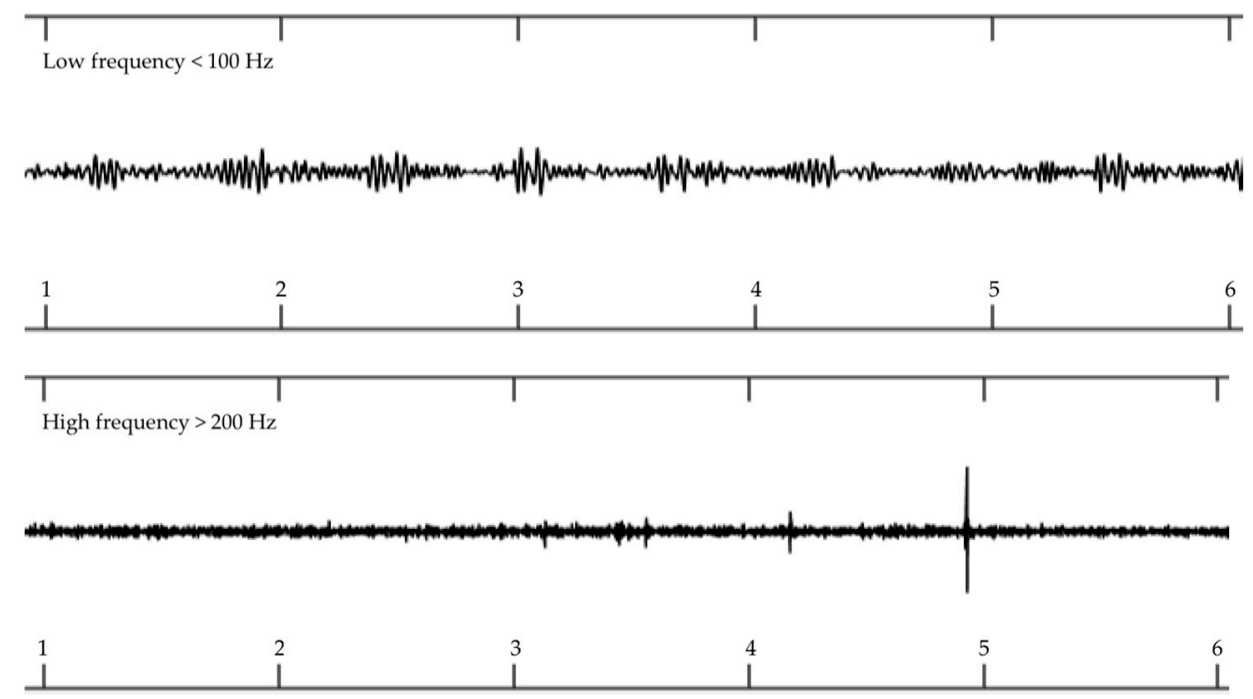

Figure 4. Example of a no maternal arterial turbulence (MAT-) test illustrating in the upper graph a cardiac-valve frequency spectrum below $100 \mathrm{~Hz}$, and in the lower graph an arterial turbulence frequency spectrum above $200 \mathrm{~Hz}$ with no visible pulsatile pattern. The $Y$-axis shows time in seconds. The amplitude is shown in arbitrary units. 
The ultrasound examinations for EFW, UA and UtA Doppler flow measures were performed by trained clinicians (MD, authors CS and OAM and one trained nurse-midwife) using trans-abdominal ultrasound (5-2 MHz abdominal probe, Sonosite TITAN ${ }^{\circledR}$ and Sonosite Turbo ${ }^{\circledR}$, US High resolution, Sonosite, Bothell, WA, USA). Head circumference (HC), abdominal circumference (AC) and femur length (FL) were measured using standard techniques [18]. Fetal weights were estimated using the Hadlock algorithm on HC, AC and FL or on AC and FL if HC was not possible to obtain with an acceptable quality [18]. Estimations of flow velocity in the left and right UtA as well as the UA were obtained using standard techniques and color pulsed-wave Doppler ultrasound [19-22]. For the UtA flow velocity waveforms were measured at the apparent crossover with the external iliac artery [20,21]. For UA, the flow velocity waveforms were measured in free-floating loop in the absence of fetal movements [22]. Appropriate gate size, minimum insonation angle, color gain and auto tracing were used when measuring flow indices [18-22].

All EFWs and BWs were categorized according to a local sex-specific hybrid EFW and BW reference generated during a previous study conducted in the same geographical area, STOPPAM [23]. Appropriate for GA (AGA) EFW and BW was defined as weight

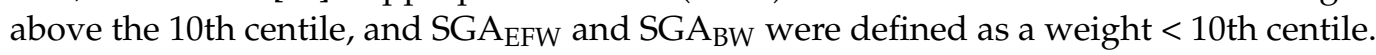
Abnormal UA and UtA Doppler flow pulsatility index (PI) were defined as PI > 95th centile [21,22]. FGR was defined as either BW < 3rd centile as a solitary biometric measure or BW $<10$ th centile and abnormal UA Doppler PI as contributory functional parameter, as approximated to the Delphi Consensus statement definition and data available [24]. The predictive performance of the biosensor was defined as a minimum of one MAT+ test during any of the three antenatal visits. Anemia was defined as hemoglobin $\leq 11 \mathrm{~g} / \mathrm{DL}$, and severe anemia was defined as hemoglobin $\leq 7 \mathrm{~g} / \mathrm{dL}$ in line with the World Health Organization's definition [25] and was measured using Sysmex KX 21N Hematological Analyzer (Sysmex Corporation, Kobe, Japan) [15].

Clinical data were entered and validated using Microsoft Office Access 2007 database (Microsoft Corporation, Redmond, WA, USA), biosensor data were filtered and visualized using Matlab R2041b (Mathworks, Natick, MA, USA) and statistical analyses were performed in SPSS version 24 (IBM, Armonk, NY, USA). Outcome (A), a solitary biometric measure (BW < 3rd centile) and outcome (B), a biometric measure and contributory functional measure (BW < 10th centile and antenatally detected UA PI > 95th centile) were investigated.

Parametrically distributed variables were expressed as mean $( \pm S D)$ and nonparametric variables were distributed as median (range). The Levene's test for homogeneity of variance was performed and parametric (Student's $t$-test), or non-parametric (MannWhitney U) test were performed where appropriate. For categorical variables, contingency table analyses and Fischer exact test with two-sided $p$-values were used, sensitivity (SE), specificity (SP), positive predictive value (PPV) and negative predictive value (NPV) according to distribution on the STOPPAM reference values [23]. Confidence interval (CI) was inferred at the 95\% level, and statistical significance at $p<0.05$.

Ethical clearance was obtained from the Medical Research Coordinating Committee of the National Institute for Medical Research (approval no. NIMR/HQ/R.8c/VOL I/394 and NIMR/HQ/R.8a/Vol. IX/1717, 6 May 2014), and written informed consent was obtained from all study participants. All procedures were performed in accordance with Good Clinical Practice adhering to the World Medical Association's Declaration of Helsinki.

\section{Results}

Among the 118 women included for investigation of the predictive performance of the biosensor, a total of 262 antenatal examinations were conducted (median 2.3 examinations per pregnancy, range 1-3). The parity was two (range 0-7), and the baseline characteristics were generally comparable to the overall FOETALforNCD cohort [15], (Table 2). 
Table 2. Clinical baseline characteristics of study participants $(n=118)$.

\begin{tabular}{cccc}
\hline Variable & Mean & Min-Max & SD \\
\hline Pregnancy length, $d$ & 280 & $259-301$ & 8 \\
Birth weight, g & 3008 & $1565-4025$ & 410 \\
Placenta weight, g & 440 & $265-867$ & 107 \\
Systolic BP & 107 & $78-151$ & 11 \\
Diastolic BP & 68 & $43-110$ & 10 \\
\hline
\end{tabular}

The number of neonates born SGA $<10$ th centile was $22(19 \%)$. Seven $(6 \%)$ newborns were FGR based in the solitary definition of BW <3rd centile [23], and one $(0.8 \%)$ neonate was born FGR using the contributory definition of BW $<10$ th centile and antenatally detected UA PI > 95th centile.

Analyses of the biosensor data showed that among the 262 independent tests, eight $(3.1 \%)$ were positive for arterial turbulence (MAT+). These eight tests were distributed among five $(4.2 \%)$ study participants.

Comparing clinical characteristics and ultrasound measures between study participants positive for arterial turbulence (MAT+) and those negative for arterial turbulence (MAT-), there was no detected difference between pregnancy length or placental weight. However, the ultrasound EFW in GA 37-39 was statistically significantly lower for the MAT+ pregnancies compared to the MAT- pregnancies $(p=0.04)$. There was a trend towards lower BW and higher systolic blood pressure among MAT+ pregnancies compared to MAT - pregnancies (Table 3). Finally, there were no differences in PI of UA and UtA between MAT+ pregnancies and MAT- pregnancies (Table 3).

Table 3. Comparison of pregnancy outcome for study participants positive for arterial turbulence (MAT+) and negative for arterial turbulence (MAT-).

\begin{tabular}{|c|c|c|c|c|}
\hline Variable & MAT $+(n=5)$ & $\begin{array}{c}\text { MAT- } \\
(n=113)\end{array}$ & 95\% CI for Difference of Means & $p$-Value \\
\hline Pregnancy length, $\mathrm{d}$ & $280 \pm 12$ & $280 \pm 8$ & -7.5 to 6.9 & $0.9^{1}$ \\
\hline $\mathrm{BW}, \mathrm{g}$ & $2689 \pm 642$ & $3022 \pm 395$ & -34.7 to 70.7 & $0.08^{1}$ \\
\hline Placenta weight, $g$ & $450 \pm 53$ & $440 \pm 108$ & -118.3 to 97.4 & $0.8^{1}$ \\
\hline Systolic BP & $117 \pm 1.3$ & $106 \pm 13$ & -22.5 to 0.5 & $0.06^{1}$ \\
\hline Diastolic BP & $77 \pm 6$ & $69 \pm 11$ & -17.5 to 0.6 & $0.1^{1}$ \\
\hline Hemoglobin, g/dL & $11.7 \pm 1.3$ & $10.8 \pm 1.4$ & -2.2 to 0.4 & $0.2^{1}$ \\
\hline EFW GA 26-28 & $981 \pm 111$ & $926 \pm 140$ & -256 to 146 & $0.6^{1}$ \\
\hline EFW GA 32-34 & $1629 \pm 265$ & $1844 \pm 267$ & -55 to 485 & $0.1^{1}$ \\
\hline EFW GA 37-39 & $2502 \pm 166$ & $2822 \pm 317$ & 3.8 to 637 & $0.04^{1}$ \\
\hline UA PI GA 26-28 & $0.95 \pm 0.3$ & $1.06 \pm 0.2$ & -0.1 to 0.3 & $0.4^{1}$ \\
\hline UA PI GA 32-34 & $0.89 \pm 0.2$ & $0.93 \pm 0.2$ & -0.2 to 0.2 & $0.7^{1}$ \\
\hline UA PI GA 37-39 & $0.99 \pm 0.2$ & $0.85 \pm 0.4$ & -0.3 to 0.02 & $0.9^{2}$ \\
\hline UtA PI GA 26-28 & $0.81 \pm 0.2$ & $0.83 \pm 0.2$ & -0.1 to 0.1 & $0.8^{1}$ \\
\hline UtA PI GA 32-34 & $0.71 \pm 0.1$ & $0.66 \pm 0.2$ & -0.1 to 0.3 & $0.4^{1}$ \\
\hline UtA PI GA 37-39 & $0.69 \pm 0.1$ & $0.68 \pm 0.1$ & -0.1 to 0.1 & $0.9^{1}$ \\
\hline
\end{tabular}

${ }^{1}$ Independent samples $t$-test, ${ }^{2}$ Mann-Whitney U test. Abbreviations: MAT, maternal arterial turbulence; CI, confidence interval; BP, blood pressure; EFW, estimated fetal weight; GA, gestational age; UA, uterine artery; PI, pulsatility index; UtA, uterine artery.

In three of the five MAT+ pregnancies, the biosensor displayed bilateral MAT+, whereas two displayed unilateral MAT+ tests. In four pregnancies, the biosensor tested positive for MAT in the early antenatal examination (GA 26-28 +/- one week). In the remaining, pregnancy inclusion was made in GA 32-34. In all but one the test-positive result occurred also in the last examination prior to time of birth.

In the one MAT+ pregnancy identified with a combination of biometric and functional parameters, BW was $1556 \mathrm{~g}$ (BW deviation was $-43 \%$ ).

When testing the biosensor data distribution frequencies using the Fischer exact test for associations, outcome (A), a solitary biometric measure (BW < 3rd centile) and outcome (B), 
a biometric measure and contributory functional measure (BW $<10$ th centile and antenatally detected UA PI $>95$ th centile) both were statistically significant (Tables 4 and 5). The sensitivity of the biosensor detecting FGR using the solitary biometric measure (BW $<3$ rd centile) was poor $(0.29)$, with specificity of $0.97(p=0.02)$, (Table 4$)$. However, when the biosensor was tested for identification of FGR defined as the contributory functional measure (BW $<10$ th centile and antenatally detected UA PI $>95$ th centile), the sensitivity was 1.00 , the specificity was 0.97 and the association was significant $(p=0.04)$, (Table 5).

Table 4. Contingency table of biosensor detection of fetal growth restriction (FGR) defined as BW $<3$ rd centile.

\begin{tabular}{cccc}
\hline Biosensor Test & Condition Positive & Condition Negative & PPV and NPV \\
\hline Test outcome positive $^{1}$ & $\mathrm{TP}=2$ & $\mathrm{FN}=3$ & $2 /(2+3)=0.40$ \\
& $\mathrm{FP}=5$ & $\mathrm{TN}=108$ & $108 /(108+5)=0.96$ \\
\hline Sensitivity & $2(2+5)=0.29$ & & \\
\hline Specificity & & $108 /(108+3)=0.97$ \\
\hline${ }^{1} p=0.02$.
\end{tabular}

Table 5. Contingency table of ultrasound detection of FGR defined as BW $<10$ th centile and antenatally detected abnormal UA PI > 95th centile.

\begin{tabular}{cccc}
\hline Biosensor Test & Condition Positive & Condition Negative & PPV and NPV \\
\hline Test outcome positive $^{1}$ & $\mathrm{TP}=1$ & $\mathrm{FN}=4$ & $1 /(1+4)=0.20$ \\
& $\mathrm{FP}=0$ & $\mathrm{TN}=113$ & $113 /(113+0)=1.00$ \\
\hline Sensitivity & $1(1+0)=1.00$ & & \\
\hline Specificity & & $113 /(113+4)=0.97$ & \\
\hline${ }^{1} p=0.04$. & &
\end{tabular}

\section{Discussion}

This study presents an innovative strategy of improving efforts in screening for FGR that is independent of the availability of clinical expertise and of setting. The use of biosensors for monitoring various health conditions opens up for easy access to viable information, as in this case for identification of arterial turbulence as marker of placental dysfunction and secondary FGR.

It is increasingly recognized that improved clinical management of FGR is urgent as current models of screening lack efficacy and only between $31 \%$ and $38 \%$ of pregnancies are identified before birth, notably in high income settings with available antenatal care programs and selective or universal ultrasonography monitoring [26,27]. In this perspective, the predictive performance of the biosensor should be assessed as a potential future method embracing several current challenges. Firstly, use of the biosensor addresses an aspect of placental dysfunction inaccessible for clinical examination until now-a direct marker of rheological change understood to link insufficient remodeling of the spiral arteries to arterial turbulence and FGR [11-14]. Secondly, it is not limited by expert handling as is the use of obstetric ultrasound, and thirdly, the technological advances of the biosensor integrate physiological data ready for various objective and artificial intelligent interpretation models allowing for monitoring irrespective of setting. Especially, in rural or low-resource settings where limited healthcare infrastructures affect clinical management the implications of biosensors are of great potential.

From this study of a cohort of 118 multiparous women with singleton- and ultrasounddated pregnancies, we showed that the double-blinded assessment of acoustic data from a wearable biosensor identified approximately one in three infants born with a BW of less than the 3rd centile in a low-resource sub-Saharan setting. Additionally, the only pregnancy complicated with BW below the 10th centile and antenatally detected abnormal UA PI was 
detected. In this case, the neonate was delivered at GA $37+0$ with BW deviation of $43 \%$ (BW $1556 \mathrm{~g}$ ) and bilateral MAT+ detected in pregnancy weeks 26-28 +/ - one week.

We defined FGR as either BW below the 3rd centile or as BW below the 10th centile and antenatally detected abnormal UA PI as different indices as earlier work showed high correlation between the markers of arterial turbulence. In addition, the Delphi Consensus statement on FGR definition points to UA PI as a functional parameter as a contributory factor to fetal weight $[7,8]$. This approach also showed increased predictive performance in this cohort $(100 \%)$ when compared to prediction of low weight only $(29 \%)$. By contrast, the specificity was the same regardless of FGR definition (97\%).

Our results showed that when adequate pregnancy-dating [16,17], adequate populationbased weight references [23], adequately trained sonographers and technology were available the biosensor test was significantly correlated with EFWs in pregnancy weeks 37-39 (Table 3). In addition, a positive trend was seen towards significant associations between the MAT test and systolic BP (Table 3). These findings imply that hypertension or preeclampsia, that share the same phenotypic pathway of placental dysfunction as FGR [12,14], could also be related to MAT as indicated in earlier case studies [28]. Consensus is stated by Khong et al. [14], that high velocity maternal malperfusion of the placenta manifesting in FGR may both cause damage to placentation and later function, and that earlier concepts and definition of maternal vascular underperfusion should be changed to maternal vascular malperfusion. It is also pointed out that a multidisciplinary approach of investigating placental function could be a way forward [28]. The present study confirms that biosensors indeed could contribute to a way forward.

Future research should be focused on sufficiently powered clinical studies as this study has a very limited sample size with only one study participant having $\mathrm{BW}<10$ th centile and UA PI $>95$ th centile $(0.8 \%)$ and seven $(6 \%)$ participants having BW $<3$ rd centile. In addition, automated quantification and interpretation of the biosensor data for machine learning to meet requirements for real-time data analysis and feedback to the user, and importantly the biosensor data, should be investigated for morphological changes related to placental dysfunction.

\section{Conclusions}

Conclusively, the pilot-study results show future potential for biosensors as screening modality for FGR in a low-resource setting, especially when the FGR definition includes both a biometrical and functional parameter.

Author Contributions: Conceptualization, C.S., B.S., M.H., D.R.; methodology, A.J., C.S., B.S., M.H., D.R.; validation, A.J., C.S., S.L.M. and O.A.M.; formal analysis, A.J. and D.R.; resources, C.S., D.T.R.M., and J.P.A.L.; writing-original draft preparation, A.J. and D.R.; writing-review and editing, C.S., B.S., K.N., B.B.N., S.L.M., D.T.R.M., J.P.A.L., M.H., O.A.M.; visualization, A.J., D.R.; supervision, C.S., B.S. and M.H.; project administration, J.P.A.L., C.S., D.T.R.M.; funding acquisition, C.S., D.R. All authors have read and agreed to the published version of the manuscript.

Funding: This research was funded by THE DANISH COUNSIL FOR STRATEGIC RESEARCH, INNOVATION FUND DENMARK, grant number 1309-0003B through support to the FOETALforNCD study, and by VIEWCARE A/S by providing biosensor technology and data architecture.

Institutional Review Board Statement: The study was conducted according to the guidelines of the Declaration of Helsinki, and approved by the Institutional Review Board of Medical Research Coordinating Committee of the National Institute for Medical Research (approval no. NIMR/HQ/R.8c/VOL I/394 and NIMR/HQ/R.8a/Vol. IX/1717, 6 May 2014).

Informed Consent Statement: Informed consent was obtained from all subjects involved in the study.

Data Availability Statement: The data presented in this study are available on request form the corresponding author. The data are not publicly available due to ethical restrictions. 
Acknowledgments: We are grateful to the study participants and the local community leaders in Korogwe and Handeni District, Tanga Region, Tanzania. We are also grateful to the research team in Tanzania-including physicians, clinical officers, nurses, laboratory technicians, data entry clerks, data manager, project drivers, and field workers for their hard work. In addition, we acknowledge the hard work and efforts provided by technicians and data managers supplying data architecture and technology from Viewcare A/S, Copenhagen, Denmark and Department of Health Science and Technology, Aalborg University, Aalborg, Denmark. Finally, we are grateful to the administration at the National Institute for Medical Research Tanga Center, Korogwe Research Station for their support.

Conflicts of Interest: Author D.R. is partly employed by Viewcare A/S. None of the other authors declare conflicts of interest. The funders had no role in the design of the study; in the collection, analyses, or interpretation of data; in the writing of the manuscript, or in the decision to publish the results.

\section{References}

1. Pels, A.; Beune, I.M.; Van Wassenaer-Leemhuis, A.G.; Limpens, J.; Ganzevoort, W. Early-onset fetal growth restriction: A systematic review on mortality and morbidity. Acta Obstet. Gynecol. Scand. 2020, 99, 153-166. [CrossRef] [PubMed]

2. Gardosi, J.; Madurasinghe, V.; Williams, M.; Malik, A.; Francis, A. Maternal and fetal risk factors for stillbirth: Population-based study. BMJ 2013, 68, 329-331. [CrossRef]

3. Crispi, F.; Miranda, J.; Gratacós, E. Longterm cardiovascular consequences of fetal growth restriction: Biology, clinical implications, and opportunities for prevention of adult disease. Am. J. Obstet. Gynecol. 2018, 218, 869-879. [CrossRef]

4. Kingdom, J.C.; Audette, M.C.; Hobson, S.R.; Windrim, R.C.; Morgen, E. A placenta clinic approach to the diagnosis and management of fetal growth restriction. Am. J. Obstet. Gynecol. 2018, 218, S803-S817. [CrossRef]

5. Ghamari, M.; Janko, B.; Sherratt, R.S.; Harwin, W.; Piechocki, R.J.; Soltanpur, C. A Survey on Wireless Body Area Networks for eHealthcare Systems in Residential Environments. Sensors 2016, 16, 831. [CrossRef]

6. Riknagel, D.; Farlie, R.; Hedegaard, M.; Humaidan, P.; Struijk, J.J. Association between maternal vascular murmur and the small-for-gestational-age fetus with abnormal umbilical artery Doppler flow. Int. J. Gynecol. Obstet. 2017, 139, 211-216. [CrossRef] [PubMed]

7. Riknagel, D.; Zimmermann, H.; Farlie, R.; Hammershøi, D.; E Schmidt, S.; Hedegaard, M.; Humaidan, P.; Struijk, J.J. Separation and characterization of maternal cardiac and vascular sounds in the third trimester of pregnancy. Int. J. Gynecol. Obstet. 2017, 137, 253-259. [CrossRef] [PubMed]

8. Riknagel, D.; Dinesen, B.; Zimmermann, H.; Farlie, R.; Schmidt, S.E.; Toft, E.; Struijk, J.J. Digital auscultation of the uterine artery: A measure of uteroplacental perfusion. Physiol. Meas. 2016, 37, 1163-1171. [CrossRef] [PubMed]

9. Schmidt, S.E.; Holst-Hansen, C.; Hansen, J.; Toft, E.; Struijk, J.J. Acoustic Features for the Identification of Coronary Artery Disease. IEEE Trans. Biomed. Eng. 2015, 62, 2611-2619. [CrossRef]

10. Boron, W.F.; Boulpaep, E.L. Medical Physiology: A Cellular and Molecular Approach, 2nd ed.; Saunders, P.A., Ed.; Elsevier: Philadelphia, PA, USA, 2012; Volume xiii, p. 1337.

11. Roth, C.J.; Haeussner, E.; Ruebelmann, T.; Koch, F.V.; Schmitz, C.; Frank, H.-G.; Wall, W.A. Dynamic modeling of uteroplacental blood flow in IUGR indicates vortices and elevated pressure in the intervillous space-A pilot study. Sci. Rep. 2017, 7, 40771. [CrossRef]

12. Burton, G.; Woods, A.; Jauniaux, E.; Kingdom, J. Rheological and Physiological Consequences of Conversion of the Maternal Spiral Arteries for Uteroplacental Blood Flow during Human Pregnancy. Placenta 2009, 30, 473-482. [CrossRef] [PubMed]

13. Avagliano, L.; Bulfamante, G.P.; Morabito, A.; Marconi, A.M. Abnormal spiral artery remodelling in the decidual segment during pregnancy: From histology to clinical correlation. J. Clin. Pathol. 2011, 64, 1064-1068. [CrossRef]

14. Khong, T.Y.; Mooney, E.E.; Ariel, I.; Balmus, N.C.M.; Boyd, T.K.; Brundler, M.-A.; Derricott, H.; Evans, M.J.; Faye-Petersen, O.M.; Gillan, J.E.; et al. Sampling and Definitions of Placental Lesions: Amsterdam Placental Workshop Group Consensus Statement. Arch. Pathol. Lab. Med. 2016, 140, 698-713. [CrossRef]

15. Hjort, L.; Møller, S.L.; Minja, D.; Msemo, O.; Nielsen, B.B.; Christensen, D.L.; Theander, T.; Nielsen, K.; Larsen, L.G.; Grunnet, L.G.; et al. FOETAL for NCD_FOetal Exposure and Epidemiological Transitions: The role of Anaemia in early Life for Non-Communicable Diseases in later life: A prospective preconception study in rural Tanzania. BMJ Open 2019, 9, e024861. [CrossRef]

16. Papageorghiou, A.T.; Kennedy, S.H.; Salomon, L.J.; Ohuma, E.O.; Ismail, L.C.; Barros, F.C.; Lambert, A.; Carvalho, M.; Jaffer, Y.A.; Bertino, E.; et al. International standards for early fetal size and pregnancy dating based on ultrasound measurement of crown-rump length in the first trimester of pregnancy. Ultrasound Obstet. Gynecol. 2014, 44, 641-648. [CrossRef]

17. Papageorghiou, A.T.; Kemp, B.; Stones, W.; Ohuma, E.O.; Kennedy, S.; Purwar, M.; Salomon, L.J.; Altman, D.G.; Noble, J.A.; Bertino, E.; et al. Ultrasound-based gestational-age estimation in late pregnancy. Ultrasound Obstet. Gynecol. 2016, 48, 719-726. [CrossRef]

18. The International Fetal and Newborn Growth Consortium. INTERGROWTH 21st Ultrasound Operations Manual; University of Oxford: Oxford, UK, 2009. 
19. Nicolaides, K.H.; Rizzo, G.; Hecher, K.X. Doppler in Obstetrics—Diploma in Fetal Medicine and ISUOG Educational Series; The Fetal Medicine Foundation: London, UK, 2002.

20. Lefebvre, J.; Demers, S.; Bujold, E.; Nicolaides, K.H.; Girard, M.; Brassard, N.; Audibert, F. Comparison of two different sites of measurement for transabdominal uterine artery Doppler velocimetry at 11-13 weeks. Ultrasound Obstet. Gynecol. 2012, 40, 288-292. [CrossRef]

21. Gómez, O.; Figueras, F.; Fernández, S.; Bennasar, M.; Martínez, J.M.; Puerto, B.; Gratacós, E. Reference ranges for uterine artery mean pulsatility index at 11-41 weeks of gestation. Ultrasound Obstet. Gynecol. 2008, 32, 128-132. [CrossRef] [PubMed]

22. Parra-Cordero, M.; Lees, C.; Missfelder-Lobos, H.; Seed, P.; Harris, C. Fetal arterial and venous Doppler pulsatility index and time averaged velocity ranges. Prenat. Diagn. 2007, 27, 1251-1257. [CrossRef]

23. Schmiegelow, C.; Scheike, T.H.; Oesterholt, M.; Minja, D.; Pehrson, C.; Magistrado, P.; Lemnge, M.; Rasch, V.; Lusingu, J.; Theander, T.G.; et al. Development of a Fetal Weight Chart Using Serial Trans-Abdominal Ultrasound in an East African Population: A Longitudinal Observational Study. PLoS ONE 2012, 7, e44773. [CrossRef]

24. Gordijn, S.J.; Beune, I.M.; Thilaganathan, B.; Papageorghiou, A.; Baschat, A.A.; Baker, P.N.; Silver, R.M.; Wynia, K.; Ganzevoort, W. Consensus definition of fetal growth restriction: A Delphi procedure. Ultrasound Obstet. Gynecol. 2016, 48, 333-339. [CrossRef] [PubMed]

25. Nutrition Impact Model Group (Anemia). Global, regional, and national trends in haemoglobin concentration and prevalence of total and severe anaemia in children and pregnant and non-pregnant women for 1995-2011: A systematic analysis of population-representative data. Lancet Glob. Health 2013, 1, 16-25. [CrossRef]

26. Andreasen, L.A.; Tabor, A.; Nørgaard, L.N.; Rode, L.; Gerds, T.A.; Tolsgaard, M.G. Detection of growth-restricted fetuses during pregnancy is associated with fewer intrauterine deaths but increased adverse childhood outcomes: An observational study. BJOG Int. J. Obstet. Gynaecol. 2021, 128, 77-85. [CrossRef] [PubMed]

27. Caradeux, J.; Martinez-Portilla, R.J.; Peguero, A.; Sotiriadis, A.; Figueras, F. Diagnostic performance of third-trimester ultrasound for the prediction of late-onset fetal growth restriction: A systematic review and meta-analysis. Am. J. Obstet. Gynecol. 2019, 220, 449-459.e19. [CrossRef]

28. Riknagel, D.; Al Humaidan, P.H.; Farlie, R.; Zimmermann, H.; Ramsing, M.; Struijk, J.J. Acoustic biomarker of placental pathophysiology and adverse fetal outcome. Placenta 2015, 36, A6. [CrossRef] 